14

\title{
Электронное и программное обеспечение автоматизированного портативного статического масс-спектрометра
}

\author{
( Ю.В. Чичагов, ${ }^{1}$ А.А. Богданов, ${ }^{1}$ Д.С. Лебедев, ${ }^{1,2}$ В.Т. Коган, ${ }^{1}$ Ю.В. Тубольцев, ${ }^{1}$ А.В. Козленок, ${ }^{2}$ \\ B.C. Морошкин, ${ }^{2}$ А.В. Березина ${ }^{2}$ \\ ${ }^{1}$ Физико-технический институт им. А.Ф. Иофрфе, \\ 194021 Санкт-Петербург, Россия \\ ${ }^{2}$ Северо-Западный федеральный исследовательский медицинский центр им. В.А. Алмазова, \\ 197341 Санкт-Петербург, Россия \\ e-mail: chichagov@mail.ioffe.ru
}

(Поступило в Редакцию 17 декабря 2015 г. В окончательной редакции 8 февраля 2016 г.)

\begin{abstract}
Представлены системы электронного обеспечения малогабаритного высокочувствительного статического масс-спектрометра, а также программные и аппаратные средства, позволяющие определять следовую концентрацию газов и летучих соединений в пробах воздуха и воды в режиме реального времени. Они позволяют готовить прибор к работе, управлять процессом проведения измерений, синхронизовать этот процесс с сопутствующими измерениями, поддерживать надежную работу инструмента, автоматически обрабатывать результаты, а также визуализировать и хранить их. Разработанные программные и аппаратные средства допускают возможность проведения непрерывных измерений продолжительностью до $100 \mathrm{~h}$. Представленные средства обеспечивают возможность обслуживания инструмента персоналом, не имеющим специальной подготовки. Результаты тестирования показали, что мобильные масс-спектрометры для геофизических и медицинских исследований, оснащенные описанными системами, позволили добиться пределов определения целевых соединений до единиц $\mathrm{ppb}(\mathrm{m})$, поддерживая разрешающую способность по массе, в зависимости от решаемой задачи, до величины 250.
\end{abstract}

DOI: 10.21883/JTF.2017.01.44027.1711

\section{Введение}

При создании мобильных малогабаритных инструментов для проведения рутинных измерений в режиме реального времени в жестких условиях эксплуатации выбор масс-анализаторов ограничен статическими, квадрупольными и реже времяпролетными системами. При этом каждая из них имеет свои преимущества.

Достоинство статического масс-анализатора на постоянных магнитах объясняется рядом его особенностей: a) возможностью регистрации ряда компонентов как в режиме сканирования, так и без него, обеспечивая при этом большой динамический диапазон измерений, б) высокой надежностью работы и малыми аппаратурными погрешностями измерений, поскольку основным элементом масс-анализатора является постоянный магнит, параметры которого практически не зависят от условий эксплуатации. Статический тип масс-анализатора отличается простотой блока электроники [1], а при ограниченных требованиях к диапазону масс (в пределах $300 \mathrm{u}$ ) выбор такого типа масс-анализатора для портативного инструмента становится обоснованным [2]. При большом количестве предложений масс-анализаторов малого размера на рынке приборов, ни российская промышленность, ни зарубежные фирмы не выпускают малогабаритных мобильных автоматизированных высокочувствительных систем специальной направленности (например, для медицинской диагностической практики или бортовых геофизических исследований) невысокой стоимости и ограниченных эксплуатационных затрат.

\section{Описание. Блок электроники}

Блок электроники поддерживает работу всех систем масс-спектрометра. Он представлен на рис. 1, где показаны его основные узлы: контроллер (CPU), источник питания детекторов (DPS), источник сканирующих напряжений (MSV), система питания ионного источника (PSIS), система управления натекателем (PSInl). Bсе эти узлы выполнены в виде модулей в стандартном $19^{\prime \prime}$-крейте высотой 3U, шириной $84 \mathrm{HP}$, с печатными платами размером $100 \times 160 \mathrm{~mm}$, шириной модулей 6НР. Для управления устройствами поддержания и измерения вакуума используется стандартный модуль контроллера вакуумных насосов, управляющих реле и вакуумных датчиков TIC-200 BOC Edwards [3].

Электропитание прибора осуществляется от сети переменного тока с помощью блока питания с выходным напряжением $+24 \mathrm{~V}$ и мощностью до $50 \mathrm{~W}$. Это напряжение далее преобразуется в модулях крейта в необходимые напряжения $(+3 \mathrm{~V},+12 \mathrm{~V}, \pm 5 \mathrm{~V})$. Первичное напряжение питания $220 \mathrm{~V}, 50 \mathrm{~Hz}$ поступает в блок питания через сетевой фильтр, питая также контроллер вакуума TIC-200.

Как видно из рис. 1, контроллер CPU взаимодействует с остальными модулями через шину SPI в качестве ведущего модуля. Структура ведомых модулей показана на рис. 2 и включает в себя последовательный интерфейс с цифровым изолятором (digital isolator), четырехканальный цифроаналоговый преобразователь (DAC), четырехканальный аналоговоцифровой преобразователь (ADC) 


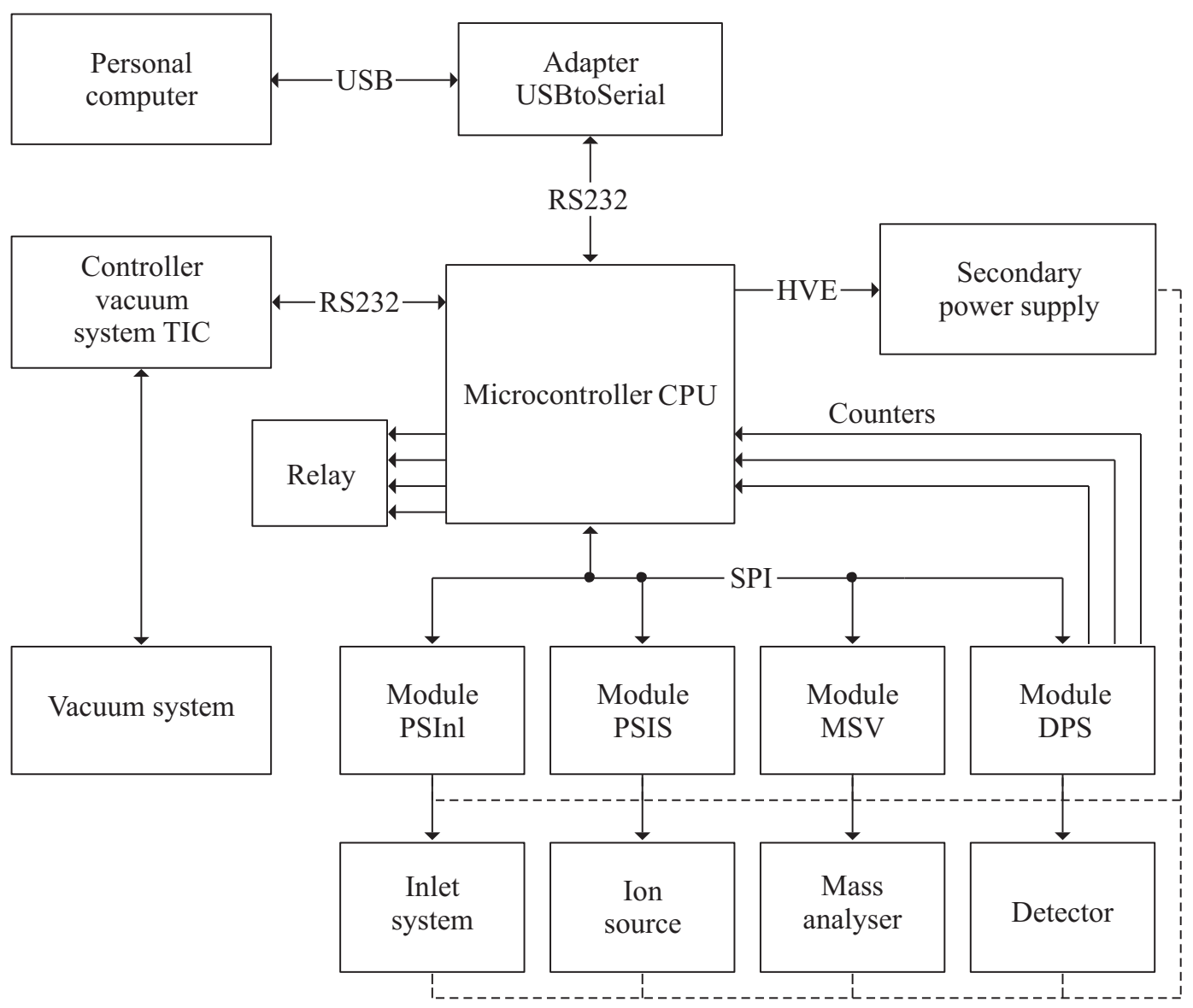

Pис. 1. Схема сопряжения модулей блока электроники с узлами портативного масс-спектрометра.

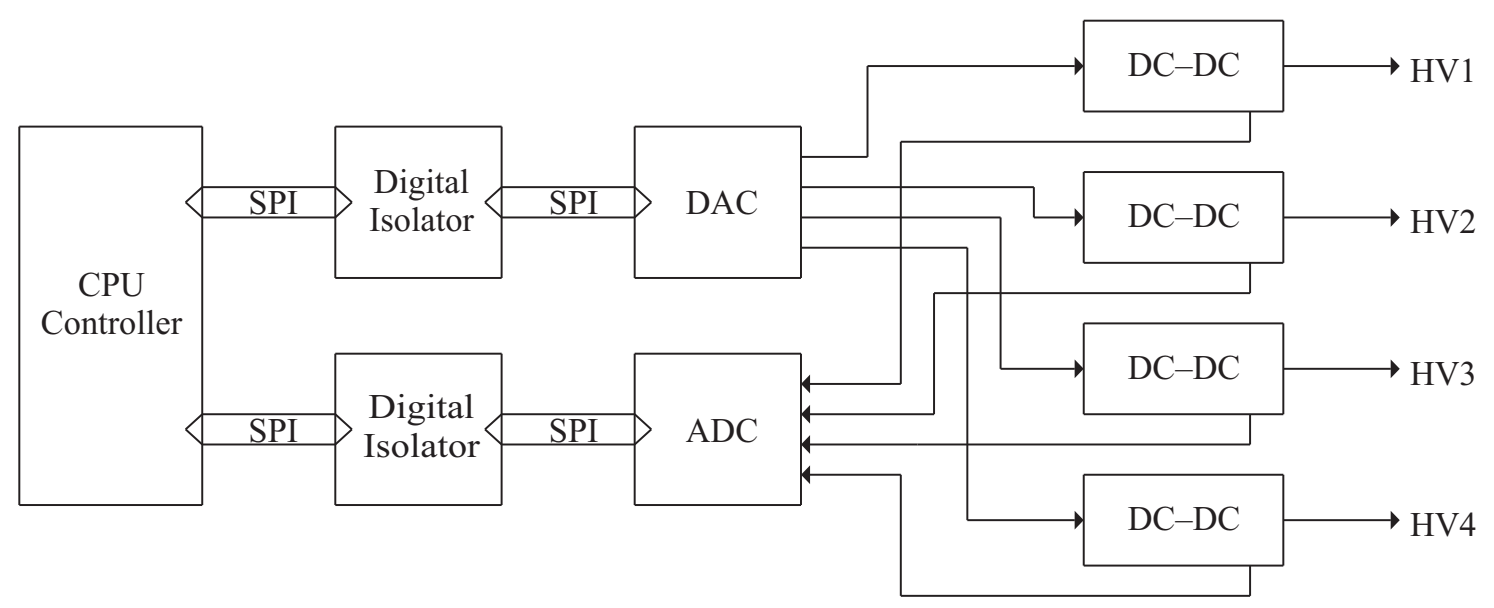

Рис. 2. Схема связи модуля CPU с ведомым модулем PSIS (MSV, DPS, SINL).

для контроля выходных напряжений, а также необходимое количество выходных высоковольтных DC-DC преобразователей, с выходов которых напряжения (HV1-4) поступают на управляющие выводы масс-спектрометра. Разрядность преобразователей DAC и ADC выбрана в соответствии с требованиями, предъявляемыми к точности соответствующих управляющих напряжений. Бо́льшая часть из них является 12-разрядными и только для DAC-модуля источника сканирующих напряжений применены два двуканальных 14-разрядных преобразователя, поскольку они задают напряжения сканирования диапазона масс и определяют повышенную точность шкалы масс при проведении измерений.

После включения блока электроники модули CPU и TIC-200 устанавливают начальные режимы работы всех остальных модулей и узлов. Управляя электромагнитны- 
ми вентилями, эти модули обеспечивают откачку вакуумной камеры масс-спектрометра форвакуумным и турбомолекулярным насосами. В ожидании необходимого рабочего уровня вакуума блок электроники блокирует работу всех высоковольтных источников.

Обмен информацией с управляющим компьютером о состоянии узлов прибора и необходимых рабочих параметрах производится через последовательный интерфейс RS-232 или USB, по выбору оператора. Чтобы понять возможности и ограничения этого процесса, необходимо рассмотреть алгоритм работы микроконтроллера CPU.

\section{Микроконтроллер. Алгоритм работы}

На рис. 3 приведена схема алгоритма работы микроконтроллера ATXMEGA32A4U_AU, который находится на плате контроллера (CPU) и координирует работу всего прибора. После включения питания микроконтроллер отрабатывает алгоритмы включения, настраивая и запуская подсистемы, после чего входит в цикл „холостого хода“", во время которого он проверяет список заданий, состоящий из двух последовательно исполняемых пунктов. Пункт 1: если есть данные от компьютера, которые нужно передать контроллеру вакуумной системы (TIC), то микроконтроллер выполняет передачу этих данных. Пункт 2: если пришла команда от оператора на включение высоких напряжений, то он исполняет алгоритм включения высоких напряжений. Далее цикл холостого хода повторяется.

Все остальные алгоритмы отрабатываются в прерываниях (они изображены на рис. 3 в виде „молний“б), которые возникают при определенных условиях и событиях. В этом случае алгоритм холостого хода прерывается, а микроконтроллер реагирует на условие или событие согласно алгоритму прерывания.

В алгоритме получения данных от компьютера участвуют подсистемы PC.USART (универсальный синхронный/асинхронный приемопередатчик) и PC.TIMER (таймep). Обмен данными между микроконтроллером и компьютером производится в асинхронном режиме. Инициировать обмен данными может как компьютер, так и микроконтроллер, что уменьшает плотность потока данных. Все основные команды имеют отклик, при помощи которого микроконтроллер сообщает компьютеру, какая именно команда была выполнена.

Пакет имеет следующую структуру: $<$ key $><$ command $>$ $[$ Data $]<$ CheckSum $><$ lock $>$, где key - байт, указывающий на начало пакета, command - байт кода команды, [Data] - массив байтов сопутствующих данных, CheckSum - контрольная сумма пакета, которая позволяет установить целостность данных, lock - байт, указывающий на конец пакета. Приведенная выше структура пакета действительна как для входящих, так и исходящих от микроконтроллера пакетов. Прием данных ограничен по времени, только для начала передачи, что предотвращает исполнение ложной команды в случае случайной помехи на шине данных, равной байту, указывающему на начало пакета (key).

Алгоритм проведения измерений осуществляется таймером реального времени (RTC) и тремя 16-битными счетчиками (A, B, C), на которые поступает сигнал о регистрации очередного зарегистрированного детекторами иона. Переполнение любого из трех счетчиков вызывает его остановку на максимальном значении. Событие RTC происходит по истечении времени измерения и вызывает особо быстрый ассемблерный код выключения всех счетчиков для обеспечения синхронности их остановки, по окончании которого микроконтроллер посылает асинхронный отчет компьютеру о завершении измерения.

В алгоритме получения данных от контроллера вакуумной системы (TIC) участвуют подсистемы TIC.USART и TIC.TIMER. Структура пакета предопределена контроллером вакуумной системы и не подразумевает ни контрольной суммы, ни байтов начала и конца пакета. Поэтому прием данных ограничен по времени для всех принимаемых байтов.

У микроконтроллера есть алгоритм контроля давления в вакуумной системе с целью предотвращения аварийного состояния прибора в целом. Поэтому подсистема TIC.TIMER имеет вторую функцию: в случае если компьютер не обращается к контроллеру TIC более $200 \mathrm{~ms}$, микроконтроллер обратится к нему сам, запросив показания датчиков давления, и в случае несоответствия или безответности запроса (предпринимаются три попытки) выключает источники высокого напряжения.

\section{Выбор режимов работы и контроль состояний блоков прибора}

После достижения необходимого рабочего уровня вакуума можно перейти к измерениям. По команде оператора сигнал разрешения включения высоковольтных узлов включает высоковольтные DC-DC-преобразователи модулей DPS, MSV, PSIS, и PSInl. После этого в модуле питания ионного источника PSIS включаются узлы преобразователей ионизирующего и двух фокусирующих напряжений, а также узел питания стабилизатора ионного тока.

Исследуемая газовая смесь проходит через систему ввода пробы, управляемую модулем PSInl, и поступает в ионный источник. Термостабилизированная мембранная система ввода пробы позволяет понижать пределы обнаружения летучих органических соединений в зависимости от способности проникновения того или иного соединения сквозь мембранный интерфейс. Летучие органические соединения и некоторые газы имеют приоритет над компонентами проб воздуха от десятков до тысяч раз. В случае прямого анализа водных проб, учитывая, что используемые полидиметилсилоксановые мембраны (SSP-100) являются гидрофобными, а молекулы рассматриваемых целевых соединений имеют невысокую степень полярности, обогащение пробы оказывается суще- 


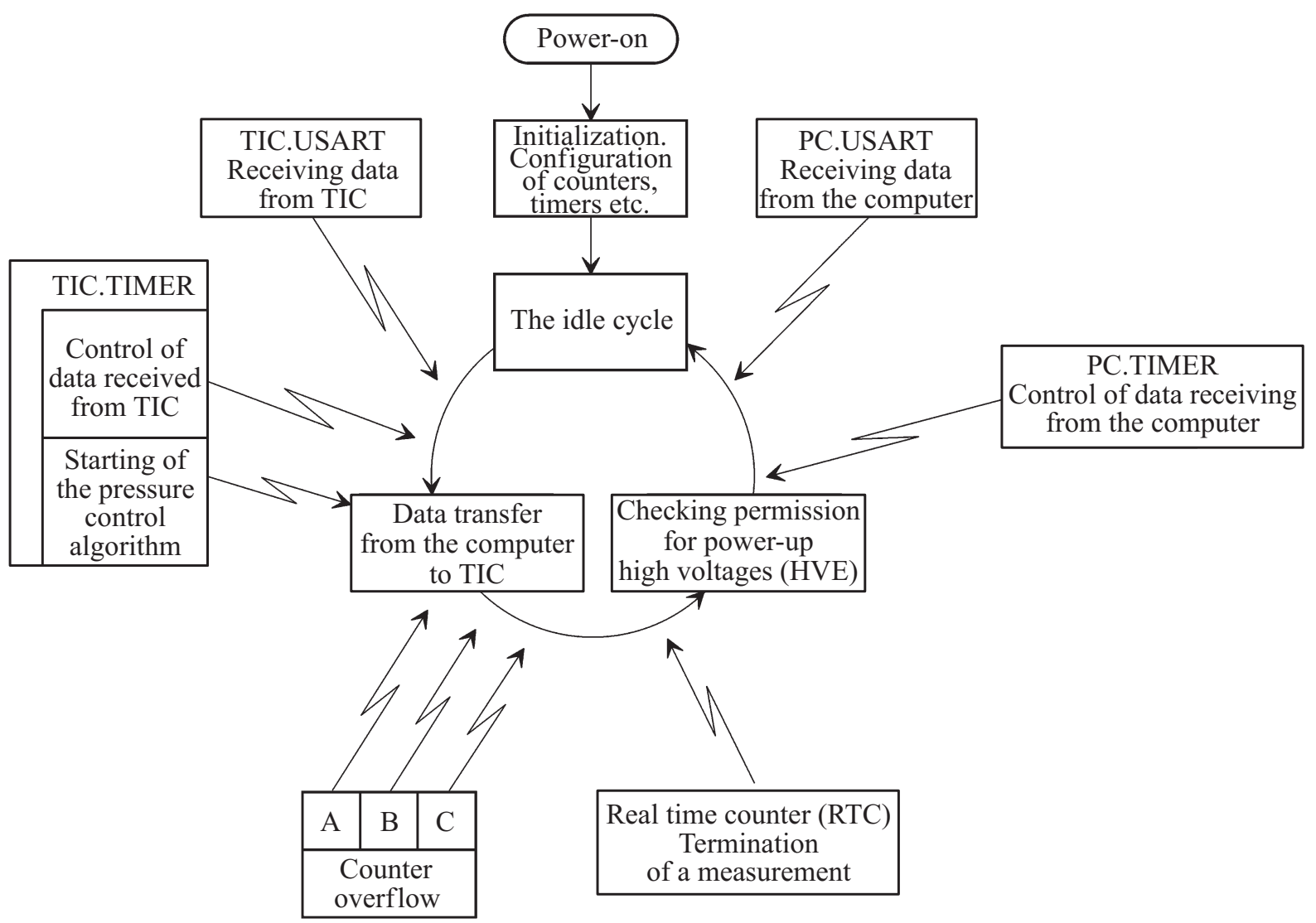

Рис. 3. Принципиальная схема работы микроконтроллера: HVE - разрешение на включение высоких напряжений; TIC - контроллер вакуумной системы; TIC.USART, PC.USART - программные модули микроконтроллера, универсальные синхронные/асинхронные приемопередатчики; TIC.TIMER, PC.TIMER - программные модули микроконтроллера, таймеры; RTC - программный модуль микроконтроллера, точный таймер реального времени; А, В, С — программные модули микроконтроллера, счетчики импульсов.

ственным и составляет величину от 1000 и более. Обогащение пробы целевыми соединениями снижает требования к динамическому диапазону масс-анализатора, даже при экспресс исследованиях (продолжительностью секунды), и позволяет ограничиться использованием канального электронного умножителя (CEM) для одновременного измерения компонентов, концентрации которых отличаются до 9 порядков величины.

В источнике ионов осуществляется ионизация пробы. Благодаря гальванической развязке выходных цепей модуля питания PSIS обеспечивается возможность подключения ионного источника к высоковольтному контакту источника сканирующего напряжения. Ионно-оптическая схема источника ионов за счет подаваемых на ее элементы напряжений формирует пучок ионов, пригодный для проведения масс-анализа. Осуществление контроля тока электронной эмиссии, обеспечивающего ионизацию молекул исследуемой пробы в источнике ионов, а также введение цепи отрицательной обратной связи по его величине, позволяет стабилизировать ионный ток на выходе источника. Значения тока эмиссии, ионизирующего, и двух фокусирующих напряжений, управляемых модулем PSIS, устанавливаются по выбору оператора.
Селекция ионов по величине отношения масса/заряд происходит после их ускорения в источнике ионов (модулем MSV) и прохождения электростатического конденсатора и постоянного секторного магнита. Получение спектра масс исследуемых смесей осуществляется сканированием ускоряющего напряжения в источнике ионов. При этом детектора (CEM) достигают ионы, массы которых находятся в однозначном соответствии с напряжением ускорения. Для уменьшения времени сканирования широкого диапазона масс используются три канала регистрации. Подробнее аналитическая часть масс-спектрометра описана в работе [2]. Сканирование диапазона масс производится путем пошагового изменения ускоряющего напряжения и пропорционального ему изменения напряжений на обкладках цилиндрического конденсатора, а также добавочного напряжения, корректирующего распределение потенциала в источнике ионов. Для этого полученный от контроллера CPU текущий код развертки преобразуется в напряжение DAC. Далее с помощью высоковольтных стабилизированных DC-DC-конверторов коды DAC трансформируются в ускоряющее и добавочное напряжения. 
Сигналы, поступающие с СЕМ в виде порции заряда, преобразуются в импульсы напряжения в усилителяхформирователях, питание которых обеспечивает модуль DPS. Затем в случае превышения порога интегрального дискриминатора они формируются по длительности и амплитуде и поступают на соответствующие входы счетчиков микроконтроллера CPU. Микроконтроллер CPU подсчитывает количество этих импульсов в трех независимых счетчиках в течение времени экспозиции, задаваемого таймером микроконтроллера, и через последовательный интерфейс передает результаты в управляющий компьютер. Величину времени экспозиции, диапазон измерения и необходимые параметры ионного источника, выбирает оператор.

В процессе измерения состава анализируемой пробы осуществляется управляемый нагрев мембранной системы ввода пробы, что повышает точность исследования [4]. Напуск пробы происходит при подаче напряжения (до $3 \mathrm{kV}$ ) на пьезозатвор системы ввода, который пропускает пробу к мембранному интерфейсу. Величину этого напряжения и температуру нагрева пьезонатекателя задает оператор через последовательный интерфейс компьютера, а затем CPU устанавливает выбранные оператором значения в модуле PSInl.

Связь всех модулей с CPU происходит по шине SPI через цифровые изоляторы, которые выполняют согласование уровней входных и выходных сигналов, а также уменьшают влияние помех (рис. 2).

Для удобства настройки прибора и контроля его текущих параметров во всех модулях имеются вспомогательные цепи, датчики напряжений и токов, сигналы которых преобразуются в коды $\mathrm{ADC}$ и через $\mathrm{CPU}$ передаются в управляющий компьютер.

В рабочем режиме для управления устройствами получения, поддержания и измерения вакуума используется модуль контроллера турбонасосов и измерительных приборов TIC-200 BOC Edwards. Он получает команды и передает информацию о вакуумных рабочих параметрах через последовательный интерфейс RS-232 транзитом через $\mathrm{CPU}$, в котором информация о наличии рабочего вакуума используется для контроля электрических параметров и защиты блоков электроники от перегрузки.

\section{Управление процедурой измерений и обработка результатов}

Для управления масс-спектрометром - контроля его рабочего состояния, проведения измерений в различных режимах, сохранения и отображения настроек и результатов измерения, их обработки и манипуляций с ними - было разработано специализированное программное обеспечение Flavor (далее - ПО). Оно, кроме того, обеспечивает расшифровку результатов, полученных в ходе длительных измерений, по заранее подготовленной библиотеке калиброванных спектров [5]. Логически и структурно оно может быть разбито на несколько малосвязанных подсистем.
Многоуровневая структура подсистемы приема-передачи сообщений (Messaging) включает в себя следующие слои абстракции: физический уровень последовательного порта (RS-232), реализация на стороне ПК описанного ранее протокола обмена со встроенным в массспектрометр микроконтроллером CPU (кодирование тела пакета, вычисление и проверка контрольной суммы). Наконец, формирование и распознавание пакетов данных обмена (по набору команда-данные) и потокобезопасную очередь с приоритетами. Причем приоритеты реализуют ограничение количества попыток отправки и таймаут для сообщений, что позволяет определять возможные проблемы связи с прибором и проводить периодическую отправку без переполнения очереди одинаковыми пакетами по таймеру запросов о состоянии прибора и значениях управляющих напряжений. Кроме того, это обеспечивает прием синхронных ответов для регистрации успешности получения команды и освобождения очереди, а также прием асинхронных аварийных сообщений со стороны микроконтроллера и их запись. Использование отдельных потоков выполнения для компонентов подсистемы обеспечивает стабильный приемотправку сообщений независимо от загруженности пользовательского интерфейса (UI).

Подсистема управления рабочим циклом ПО (Controller) отвечает за поддержание текущего состояния режима работы, загрузку необходимых для корректной работы и проведения измерений настроек, трансляцию запросов, инициируемых пользователем при помощи элементов управления в UI, в подсистему приема-передачи (Messaging). Также в ходе рабочего цикла происходит обратное предоставление пользовательскому интерфейсу вновь пришедших полученных данных о контролируемых параметрах, состоянии и результатах измерения и сигнализация UI об их обновлении для своевременного отображения оператору.

Важнейшими компонентами рабочего цикла являются режимы измерения (подсистема Measuring). Они включают в себя законы преобразования логических ступеней измерения в физические ступени напряжения и организацию нескольких режимов измерения. Режим „сканирования“ позволяет, наряду с возможностью детального рассмотрения спектра, проводить ускоренный его просмотр. Прецизионный режим служит для более тщательного изучения отдельных участков спектра (до 20 участков с разной экспозицией на 1 ступень измерения). Режим „мониторинга“, представляющий циклично повторяющийся режим прецизионного измерения, формирует интегральные данные на каждой итерации.

При необходимости определения вклада отдельных соединений в исследуемую смесь известного качественного состава предварительно готовится библиотека относительного вклада характерных пиков спектров чистых соединений, предположительно присутствующих в смеси (подсистема Library). Затем из них формируется „идентификационная“ матрица (таблица), позволяющая после каждой итерации режима мониторинга определять 
Идентификационная матрица. Интенсивности пиков масс-спектров целевых соединений при концентрации 100 ррь в пробе

\begin{tabular}{l|c|c|c|c|c}
\hline \multirow{2}{*}{ Соединение } & \multicolumn{5}{|c}{ Молярная масса, u } \\
\cline { 2 - 6 } & 15 & 30 & 39 & 42 & 58 \\
\hline метан & $135 ; \pm 12$ & - & - & - & - \\
этан & 10 & $5530 ; \pm 75$ & - & 990 & - \\
пропан & 3 & 360 & $3200 ; \pm 130$ & $5200 ; \pm 118$ & 170 \\
изобутан & 7 & 180 & 3100 & 3520 & $1980 ; \pm 45$ \\
н-бутан & 10 & 250 & 3990 &
\end{tabular}

концентрации конкретных веществ в пробе в режиме реального времени. Для ускорения вычислений перед началом цикла измерений производится предварительное LU-разложение сформированной матрицы.

Внутренние структуры данных приложения (сгруппированные в подсистему Data) позволяют организовать хранение текущих настроек, контролируемых параметров, результатов в процессе измерения, загруженных для отображения спектров.

Модуль настроек (Settings) обеспечивает сериализацию (преобразование) структур данных в формат XML или текстовый построчный вывод (при длительных режимах измерения) для последующего удобства экспортирования в табличный формат, и их обратное преобразование, десериализацию, (восстановление начального состояния структуры данных) для отображения в UI по запросу оператора. Он также предоставляет возможность подсистемам ПО проводить разнообразные процедуры манипуляции с данными (вычитание спектров одного типа и полученных при одинаковом режиме измерения, их нормировку).

Пользовательский интерфейс (UI) при взаимодействии с подсистемами Controller, Settings и Data обеспечивает вывод на экран контролируемых параметров, сообщений об ошибках, результатов текущих измерений, а также предоставляет оператору диалоги модификации разнообразных настроек. Кроме того, он содержит элементы управления масс-спектрометром (формирующие при их активации запросы на действие в системе рабочего цикла ПО) и запуска/досрочного прерывания циклов измерения.

\section{Результаты тестирования мобильного масс-спектрометра для геофизических и медицинских исследований}

Оснащение портативного масс-спектрометра описанным в работе обеспечением позволило адаптировать инструмент к in situ, on-line исследованиям, проводимым в области медицинского и геофизического мониторинга газов и летучих веществ.

Результаты испытаний масс-спектрометра для диагностики патологий в медицине [2] представлены на рис. 4. Фрагмент масс спектра гексафторида серы на рис. 4, $a$ получен в режиме непрерывного сканирования диапазона масс. Поскольку сканирование по массе в обсуждаемом приборе проводится изменением ускоряющего напряжения при относительно малых его значениях (до $1500 \mathrm{~V}$ ), что приводит к небольшим отличиям в соотношениях пиков от стандартных, рядом представлен фрагмент масс-спектра этого же соединения из каталога NIST для масс-спектров с электронной ионизацией. На рис. $4, b$ показаны результаты мониторинга эталонной воздушной смеси с дискретно меняющимся количеством ацетона. Здесь же проведено сопоставление аргонакомпонента, который не участвует в химии процессов дыхания и, являясь естественным репером в воздушной смеси, служит только для контроля процесса измерения, и ацетона, являющегося продуктом процессов метаболизма, который позволяет контролировать реакцию организма здорового или больного человека на внешние воздействия. Близкие интенсивности этих соединений на графике при существенном различии их концентрации в исследуемой смеси объясняются различием ряда характеристик и условий: сечения ионизации аргона $\left(3 \cdot 10^{-16} \mathrm{~cm}^{2}\right)$ и ацетона $\left(10^{-15} \mathrm{~cm}^{2}\right)$, долей характерного пика в спектре масс-аргона $(0.8$ для $40 \mathrm{u})$ и ацетона $(0.25$ для $58 \mathrm{u})$, отношением проникающих способностей ацетона и аргона сквозь материал мембраны ( 18), отношением экспозиции детектора на одной точке ( 17 указано на рис. 4$)$, отношением количества циклов в одном измерении аргона и ацетона 1/3. На рис. 4, с показаны результаты изменений концентрации ацетона в выдыхаемом человеком воздухе. Изучение характера изменения концентрации ацетона в процессе дыхания и позволяет сделать заключение о предрасположенности наблюдаемого к той или иной патологии. Мониторинг выдыхаемого воздуха проводился в соответствии со стандартной процедурой. Интервал (1-2) соответствует подаче на вход масс-спектрометра выдыхаемого воздуха при отсутствии какого-либо воздействия на испытуемого. Интервал $(2-3)$ отражает динамику содержания ацетона в выдыхаемом воздухе во время теста с постоянно возрастающей физической нагрузкой (с мощностью, допустимой для определенной группы испытуемых). Интервал (3-4) соответствует прекращению нагрузки с продолжением газоанализа выдыхаемого воздуха в восстановительном периоде. Затем останавливается тест (4) и прекращается подача пробы в прибор (5). 

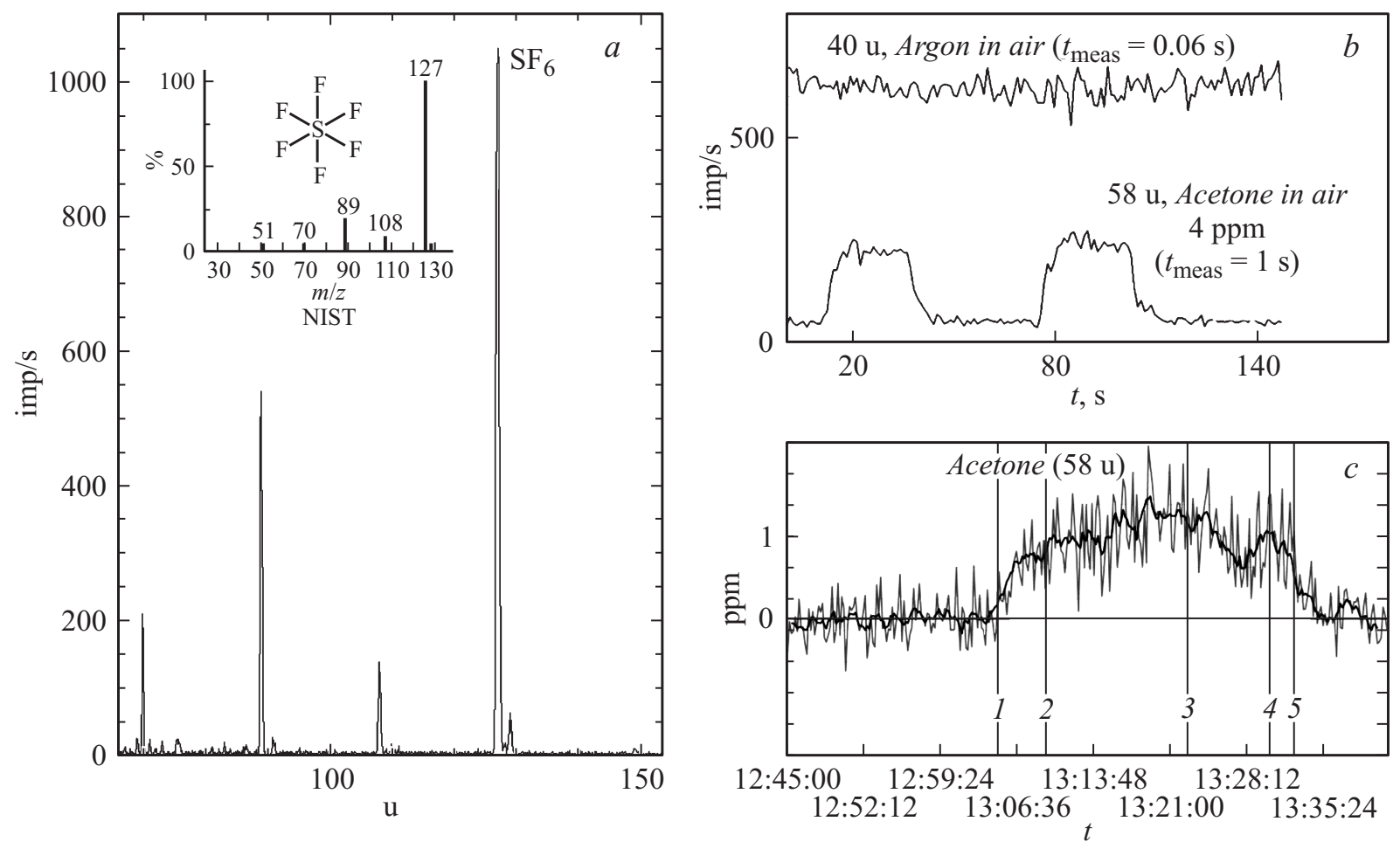

Рис. 4. Результаты испытаний масс-спектрометра: $a-$ фрагмент масс-спектра гексафторида серы, $b-$ воздушная смесь с дискретно меняющимся количеством ацетона, $c$ - изменение концентрации ацетона в выдыхаемом воздухе при нагрузочном тестировании здорового человека. Значение концентрации приведено в единицах $\operatorname{ppm}(\mathrm{m})$, т. е. в миллионных долях массы целевого соединения (ацетона) по отношению к массе пробы воздуха в единице объема.
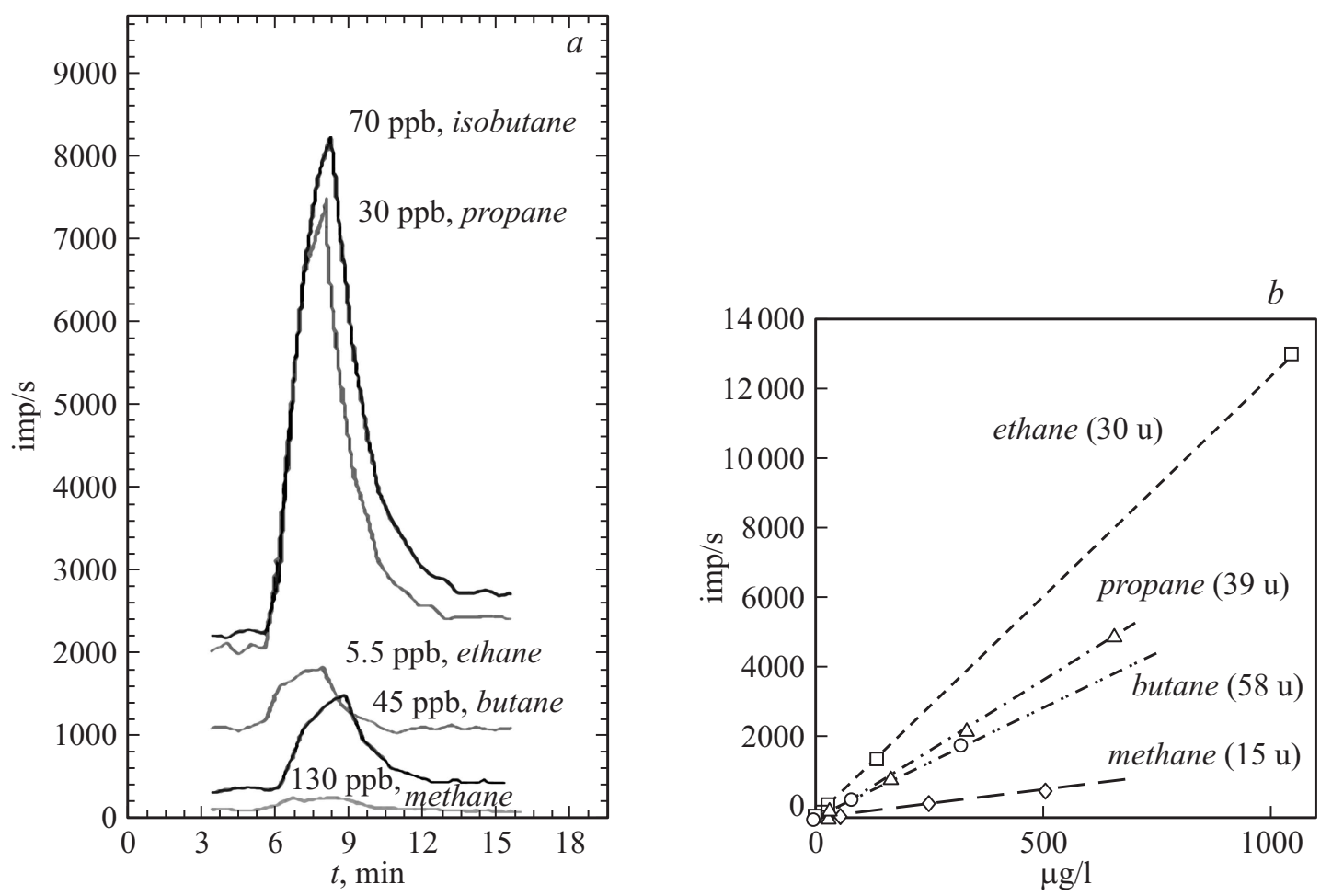

Рис. 5. Результаты испытаний масс-спектрометра для геофизических исследований: $a-$ фрагмент непрерывных наблюдений за характерными пиками растворенных в воде газов. Концентрации целевых соединений метана, этана, пропана, бутана и изобутана изменялись в процессе измерения от фоновых величин до значений, указанных на графике, $b$ - зависимость отклика инструмента для целевых газов от их концентрации в водной пробе. Значение концентрации приведено в единицах $\mathrm{ppb}(\mathrm{m})$, т. е. в миллиардных долях массы целевых соединений по отношению к массе пробы воды в единице объема. 
Подготовка и испытание инструмента предназначенного для геофизического мониторинга растворенных в воде газов описана в работе [6]. На рис. 5, a показаны данные контроля интенсивностей характерных пиков масс-ионов целевых соединений, полученные в процессе последовательно проводимых циклов их сканирования (допускают продолжительные измерения, проводимые в автоматизированном режиме, описанном ранее как режим „мониторинг“). Выбор продолжительности каждого цикла составляет $\sim 40 \mathrm{~s}$ и ограничен, с одной стороны, требуемой чувствительностью и временем отклика мембраны на изменение концентрации в пробе (при прямом анализе водных проб, по соображениям прочности мембран, допустимо использовать материал толщиной $\sim 0.3 \mathrm{~mm}$, для которого минимальный отклик по метану составляет $30 \mathrm{~s}$ [4]). А с другой стороны, продолжительность каждого цикла ограничивается требованиями к быстродействию инструмента, допускающими время реакции прибора в пределах 1 min (прямым назначением прибора для анализа водных проб является разведка залежей нефти и газа на шельфе северных морей в бортовых исследованиях [4]). Поскольку связь между концентрацией соединения в пробе и величиной отклика прибора в интервале от 0 до $0.5-1.0 \mu \mathrm{g} / 1$ имеет линейный характер (рис. $5, b$ ), определение концентрации может проводиться автоматически в процессе измерения с использованием представленной в таблице идентификационной матрицы.

Эта матрица составлена по результатам калибровочных измерений характерных пиков масс целевых соединений в бинарных смесях (вода-метан, вода-этан, вода-пропан, вода-бутан) с известной концентрацией $100 \mathrm{ppb}$, которая и указана в заголовке таблицы.

На рис. 5, $а$ приведены концентрации смесей сложного состава, содержащих группы целевых соединений различной концентрации, рассчитанные на момент достижения максимальных значений их интенсивностей (оценка концентраций проводится на каждый момент достижения очередного максимума на графике интенсивностей сигналов для $k$-характерных пиков целевых соединений решением системы $k$-уравнений, в которых используются коэффициенты идентификационной матрицы). При этом делается допущение, что учитываемые матрицей соединения являются существенно преобладающими над остальными, что, как правило, выполняется вблизи мест просачивания известных летучих соединений из месторождения. Следует иметь ввиду, что результаты мониторинга in situ, on-line, полученные с применением настоящего прибора, являются полуколичественными и оценочными. После обнаружения избытка какого-либо из целевых соединений принимается решение о проведении более тщательных измерений с применением описанного инструмента в режиме длительной подачи пробы от выбранного источника. При необходимости и доступности более точных стационарных инструментов возможно их применение после доставки проб в лабораторию.

\section{Заключение}

Необходимость создания малогабаритных инструментов, предназначенных для многокомпонентного анализа следовых количеств летучих соединений и газов в режиме реального времени в пробах воды и воздуха, объясняется отсутствием их промышленного производства. Чрезвычайно важную роль в составе этих инструментов играет электронное и программное обеспечение, один из путей создания которого, а также его реализация описаны в настоящей работе. Выбор масс-спектрометра, работающего в статическом режиме, позволил остановиться на сравнительно простых, компактных и надежных электронных схемах, обеспечивающих высокую стабильность и точность работы прибора. Управление состояниями систем масс-спектрометра, контроль электрических параметров и связь с компьютером осуществляется микроконтроллером по описанному в работе алгоритму. Процесс масс-спектрометрического измерения проводится с помощью авторских программ с использованием обеспечения Flavor, позволяющих автоматизировать проведение экспрессного многокомпонентного анализа летучих соединений низкой концентрации в пробах воды и воздуха в режиме реального времени.

В настоящей работе представлены результаты измерений, полученные портативными масс-спектрометрами, оснащенными электронным и программным обеспечением, описанным выше. Тестовые испытания двух изготовленных приборов показывают возможность их широкого применения в медицинской диагностике, а также при геофизической разведке полезных ископаемых.

\section{Список литературы}

[1] Badman E.R., Cooks R.G. // J. Mass Spectrom. 2000. Vol. 35. P. 659-671.

[2] Коган В.Т., Козленок А.В., Чичагов Ю,В., Антонов А.С., Лебедев Д.С., Богданов А.А., Морошкин В.С., Березина А.В., Викторова-Леклерк О.С., Власов С.А., Тубольщев Ю.В. // ЖТФ. 2015. Т. 85. Вып. 10. С. 135-140.

[3] Электронный ресурс. Режим доступа: www.edvac.pl/artykulPlik/ file_254.pdf

[4] Коган В.Т., Антонов А.С., Лебедев Д.С., Козленок А.В., Власов С.Ю., Чичагов Ю.В., Викторов И.В. // ПТЭ, 2013. T. 4. C. $112-116$.

[5] Коган В.Т., Лебедев Д.С. Научно-исследовательская лабораторная работа. Портативный масс-спектрометр для прямого контроля газов и летучих соединений в пробах воздуха и воды. Издат. СПбГПУ. 2012.

[6] Коган В.Т., Антонов А.С., Лебедев Д.С., Власов С.А., Краснюк А.Д. // ЖТФ. 2013. Т. 83. Вып. 3. С. 132-139. 